\title{
PEIXES RECIFAIS DA COSTA DA PARAÍBA, BRASIL
}

\author{
Luiz A. Rocha ${ }^{1}$ \\ lerecê L. Rosa ${ }^{1}$ \\ Ricardo S. Rosa ${ }^{1}$
}

\begin{abstract}
ReEf fishes from Paraíba, Brazil. The coral reef is an ecossystem which evokes great interest, for its beauty, species diversity and fisheries potential. The coast of Paraiba State, northeast Brazil, possesses several natural reefs and shipwrecks which are inhabited by a rich fish fauna. In order to survey the reef fish fauna of Paraíba State, a total of 118 dives, utilizing SCUBA diving techniques, were carried out in 27 sampling points (depths: 0,5-66m), from February/1995 to May/1996. Specimens were collected whenever underwater identification was precluded. A total of 157 known species belonging to 59 families were found. Four unidentified species, one dasyatid, one haemulid, one scarid, and one labrid were recorded. Haemulidae was the most representative family in number of individuals, whereas Carangidae was the most speciose family. This paper is part of a broader study of the northeast Brazilian reef fishes.
\end{abstract}

KEY WORDS. Brazil, Paraiba, reef fishes

O recife de coral é um ecossistema extremamente rico, produtivo e diversificado, e faz parte de um sistema tropical costeiro, do qual muitas pessoas dependem. Ele fornece alimento à população, é uma notável fonte de emprego e renda (dos setores pesqueiro e turístico), protege fisicamente as praias tropicais, serve como habitat para muitas espécies e oferece materiais únicos para educação e pesquisa científica (WHITE et al. 1994). Nesses locais os peixes alcançam grande diversidade (WELls \& HANNA 1992), encontrando-se mais de 4000 espécies nos recifes do Indo-Pacífico e cerca de 400 nos do Caribe (SALE et al. 1994). Além dessa riqueza taxonômica, observa-se também uma enorme diversidade de formas, hábitos, comportamentos e relações (SALE 1991).

Ecossistemas com composição faunística similar àquela dos recifes de coral são formados por navios naufragados, sendo comum a adoção do termo "recifes artificiais" para designar tais locais (POTTS \& HUlBerT 1994). De acordo com CHOU (1991) as grandes placas metálicas de naufrágios servem de substrato para os corais e algas calcárias, e as fendas e túneis formados entre os destroços dos navios oferecem abrigo a diversas espécies de peixes.

Com o desenvolvimento de equipamentos de mergulho e sistemas de transporte modernos, os recursos naturais encontrados nos recifes, naturais e artificiais, tornaram-se muito mais acessíveis aos pesquisadores e à população de um modo geral (MOYLE \& CECH 1996; BAKUS 1994) e, como não há um manejo adequado dessas áreas, a exploração desordenada das mesmas torna-se cada dia mais evidente (BREWER 1994; SALE et al. 1994).

1) Departamento de Sistemática e Ecologia, Centro de Ciências Exatas e da Natureza, Universidade Federal da Paraiba. 58059-900 João Pessoa, Paraíba, Brasil. 
Apesar de existirem alguns trabalhos publicados sobre a ictiofauna marinha do Nordeste brasileiro (STARKS 1913; RouX 1973; RosA 1980; RAMOS 1994), poucos são os dados publicados acerca da ictiofauna recifal desta área. Levantamentos ictiofaunísticos foram realizados por KOIKE \& GUEDES (1981) nos recifes de Pernambuco e estados vizinhos, FerReIRA et al. (1995) na praia de Tamandaré, Pernambuco, RosA et al. (1997) em poças de maré da praia do Cabo Branco, Paraíba e Rosa \& Moura (1997) no Atol das Rocas, Rio Grande do Norte.

O presente trabalho tem por objetivo fornecer uma lista das espécies de peixes recifais da costa da Paraíba e registrar aspectos ecológicos desta comunidade, visando ampliar o conhecimento acerca da ictiofauna recifal do Nordeste brasileiro, o que poderá subsidiar projetos de conservação e manejo destes recursos.

\section{MATERIAL E MÉTODOS}

\section{Área de estudo}

A área amostrada (Fig. 1, Tab. I) está compreendida entre as praias de Barra de Mamanguape (município de Rio Tinto - latitude $06^{\circ} 46^{\prime}$ 'S) e de Acaú (município de Pitimbú - latitude 07035'S). Segundo KEMPF (1970), a plataforma continental nesta área apresenta largura relativamente reduzida, sua maior parte encontra-se em profundidades menores que $40 \mathrm{~m}$ e o início do talude dá-se geralmente entre 50 e 60m. LEÃo (1986) descreve os recifes encontrados no Nordeste brasileiro como pequenos, alongados, com fauna pobre em corais e, em sua maioria, assentados sobre um substrato rochoso não coralino.

\section{Elaboração da lista}

Para a elaboração da lista dos peixes recifais, foram identificadas as espécies observadas durante 118 mergulhos, entre os meses de fevereiro de 1995 e maio de $1996 \mathrm{em} 20$ recifes naturais e sete artificiais ao longo da costa (Fig. 1, Tab. I), cujas profundidades variaram de 0,5 a $66 \mathrm{~m}$. Utilizou-se uma placa de PVC para anotações subaquáticas, na qual eram registradas todas as espécies vistas em cada mergulho e alguns aspectos ecológicos da comunidade de peixes.

Para a complementação da lista de espécies foi analisado material proveniente de duas coletas, uma no recife do Cabo Branco no dia 10 de julho de 1994, e outra no recife do Picãozinho no dia 19 de abril de 1996, nas quais utilizou-se ictiotóxico à base de rotenona. Espécimes também foram coletados em mergulhos, utilizando-se um puçá, quando a identificação in loco não foi possível. O material coletado foi fixado em formol a $10 \%$, preservado em álcool etílico $70 \%$, e depositado na Coleção Ictiológica da Universidade Federal da Paraíba (UFPB). Na identificação das espécies foram utilizados os guias de RANDALL (1968), MENEZES \& Figueiredo (1985), BÖHLKE \& CHAPLIN (1993), LIESKE \& MYERS (1994) e HUMANN (1995).

O material foi organizado sob a forma de lista sistemática, de acordo com NELSON (1994). Na lista de espécies, aquelas assinaladas por asterisco $(*)$ representam primeiros registros formais para a costa da Paraíba. As espécies coletadas apresentam número de coleção; as demais foram observadas, fotografadas (Figs 2, 3) e/ou filmadas. 


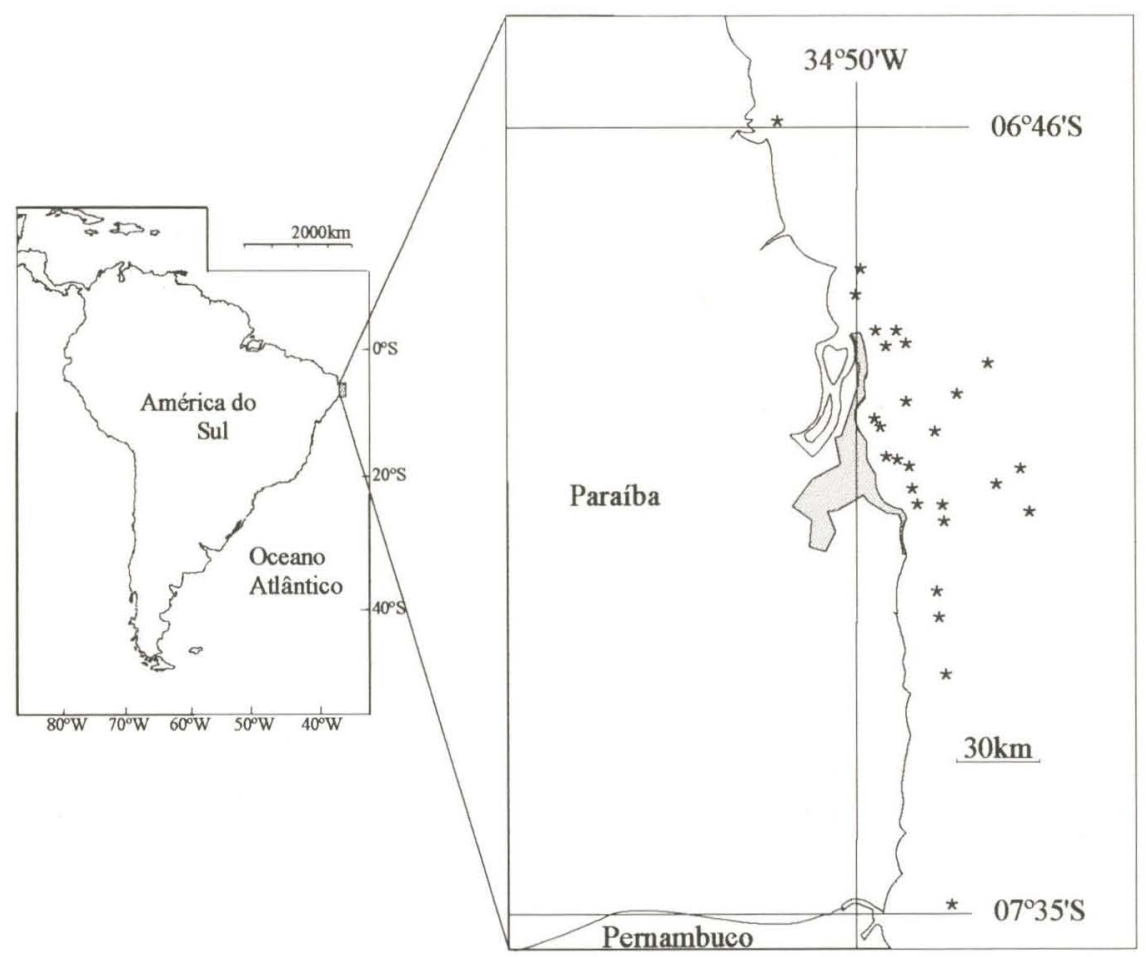

Fig. 1. Área de estudo, com indicação dos pontos visitados $\left({ }^{*}\right)$.

Tabela I. Pontos amostrais na costa da Paraiba, com indicação de profundidade, distância da costa, município, e número de mergulhos por localidade.

\begin{tabular}{lcccl}
\hline \multicolumn{1}{c}{ Local } & $\begin{array}{c}\text { Profundidade } \\
(\mathrm{m})\end{array}$ & $\begin{array}{c}\text { Distância da costa } \\
(\mathrm{Km})\end{array}$ & $\begin{array}{c}\text { Número de } \\
\text { mergulhos }\end{array}$ & Municipio \\
\hline Recifes do late & $0,5-2,0$ & 1 & 2 & Joăo Pessoa \\
Recifes de Tambaú & $0,5-2,0$ & 1 & 3 & Joắ Pessoa \\
Recifes de Formosa & $0,5-2,0$ & 2 & 3 & Cabedelo \\
Recifes da Penha & $0,5-3,0$ & $2-3$ & 4 & João Pessoa \\
Recife de Areia Vermelha & $0,5-5,0$ & 1,5 & 2 & Cabedelo \\
Recifes da Barra de Mamanguape & $0,5-6,0$ & 1 & 1 & Rio Tinto \\
Recife do Picăozinho & $0,5-6,0$ & 1,5 & 14 & Joăo Pessoa \\
Recifes da Ponta do Seixas & $0,5-6,0$ & $0,1-2,0$ & 3 & Joăo Pessoa \\
Recifes do Bessa & $0,5-6,0$ & 2 & 2 & Joăo Pessoa \\
Recife do Picăo das Agulhas & $0,5-8,0$ & 3 & 2 & Joăo Pessoa \\
Naufrágio dos Sinos & 3 & 3 & 1 & Lucena \\
Naufrágio dos Tinteiros & 5 & 1,5 & 2 & Cabedelo \\
Naufrágio do Vanadouro & 7 & 5 & 4 & Lucena \\
Recife do Caiau & $6-8$ & 3 & 2 & Joăo Pessoa \\
Recife da Pedra Nova & 10 & 4 & 2 & Conde \\
Recife da Curuba Grande & 11 & 5 & 1 & Conde \\
Recife do Taci de Cima & 12 & 5 & 5 & João Pessoa \\
Recife do Tacizinho & 12 & 6 & 2 & Conde \\
Naufrágio do Alice & 12 & 6 & 17 & Joăo Pessoa \\
& & & & (Cont.)
\end{tabular}


Tabela I. Continuação.

\begin{tabular}{lcccl}
\hline \multicolumn{1}{c}{ Local } & $\begin{array}{c}\text { Profundidade } \\
(\mathrm{m})\end{array}$ & $\begin{array}{c}\text { Distáncia da costa } \\
(\mathrm{Km})\end{array}$ & $\begin{array}{c}\text { Número de } \\
\text { mergulhos }\end{array}$ & Município \\
\hline Recife do Tarci de Cabedelo & 15 & 7 & 5 & Cabedelo \\
Naufrágio do Queimado & 18 & 9 & 23 & Joăo Pessoa \\
Naufrágio do Alvarenga & 20 & 11 & 7 & Joăo Pessoa \\
Naufrágio do Vapor Bahia & 24 & 12 & 4 & Pitimbú \\
Recife da Pedra de Baixo & 30 & 15 & 1 & Cabedelo \\
Recife do Raso de Baixo & 38 & 28 & 1 & Conde \\
Recife do Buraco & 54 & 31 & 2 & Conde \\
Recife da Parede dos Dentǒes & 66 & 35 & 3 & João Pessoa \\
\hline
\end{tabular}

\section{RESULTADOS E DISCUSSÃO}

Foram identificadas 157 espécies, distribuídas em 59 famílias. Quanto ao número de espécies, Carangidae (12 espécies) foi a família mais representativa, seguida de Serranidae (11 espécies) e Haemulidae (9 espécies). Apesar de todas as espécies identificadas estarem citadas para a Província Brasileira (sensu BRIGGS 1974), com distribuição geográfica abrangendo a costa nordestina, 69 ainda não haviam sido formalmente registradas para a costa da Paraíba. Destas, várias são capturadas para fins comerciais (pesca e aquarismo).

Dentre os táxons observados, quatro não puderam ser identificados ao nível específico e possivelmente representam novas espécies: Haemulon sp. (Haemulidae) encontrada em grandes cardumes nos recifes com profundidades variando de 2 a 30m; Scarus sp. (Scaridae) observada entre 3 e 30m de profundidade, sendo a mais freqüentemente avistada do gênero; Clepticus sp., espécie zooplanctívora, observada em cardumes na coluna d'água e Dasyatis sp., observada em fundos de areia próximo aos recifes até $15 \mathrm{~m}$ de profundidade.

Estudo anterior acerca dos peixes recifais e de áreas próximas aos recifes de Pernambuco e estados vizinhos (KOIKE \& GUEDES 1981), realizado entre 1968 e 1979, listou 74 espécies pertencentes a 42 famílias. Destas, 23 não foram encontradas no presente trabalho: Elops saurus Linnaeus, 1758, Bascanichthys teres (Goode \& Bean, 1822), Ophicthus ophis (Linnaeus, 1758), Myrophis punctatus Lutken, 1852, Lile piquitinga (Schreider \& Ribeiro, 1903), Lycengraulis grossidens (Agassiz, 1829), Anchoa lamprotaenia Hildebrand, 1943, Arius herzbergii (Bloch, 1794), Neobythites gilli Goode \& Bean, 1885, Xenomelaniris brasiliensis (Quoy \& Gaimard, 1824), Serranus baldwini (Evermann \& Marsh, 1902), Oligoplites saurus (Bloch \& Schneider, 1801), Ulaema lefroyi (Goode, 1874), Eucinostomus gula (Cuvier, 1830), Menticirrhus martinicensis (Cuvier \& Valenciennes, 1830), Stegastes leucostictus (Müller \& Troschel, 1848), Hemipteronotus novacula (Linnaeus, 1758), Doratonotus megalepis (Gunther, 1862), Nomeus gronovii (Gmelin, 1788), Syacium micrurum Ranzani, 1840, Bothus ocellatus (Agassiz, 1831), Symphurus plagusia (Bloch \& Schneider, 1801), Sphoeroides spengleri (Bloch, 1785).

Para a praia de Tamandaré, Pernambuco, FerreIRA et al. (1995) listaram 99 espécies, 14 das quais não foram observadas nos recifes da área deste estudo: Harengula clupeola (Cuvier, 1829), Synodus intermedius (Spix, 1829), Aulostomus maculatus Valenciennes, 1845, Fistularia tabacaria Linnaeus, 1758, Pseudo- 

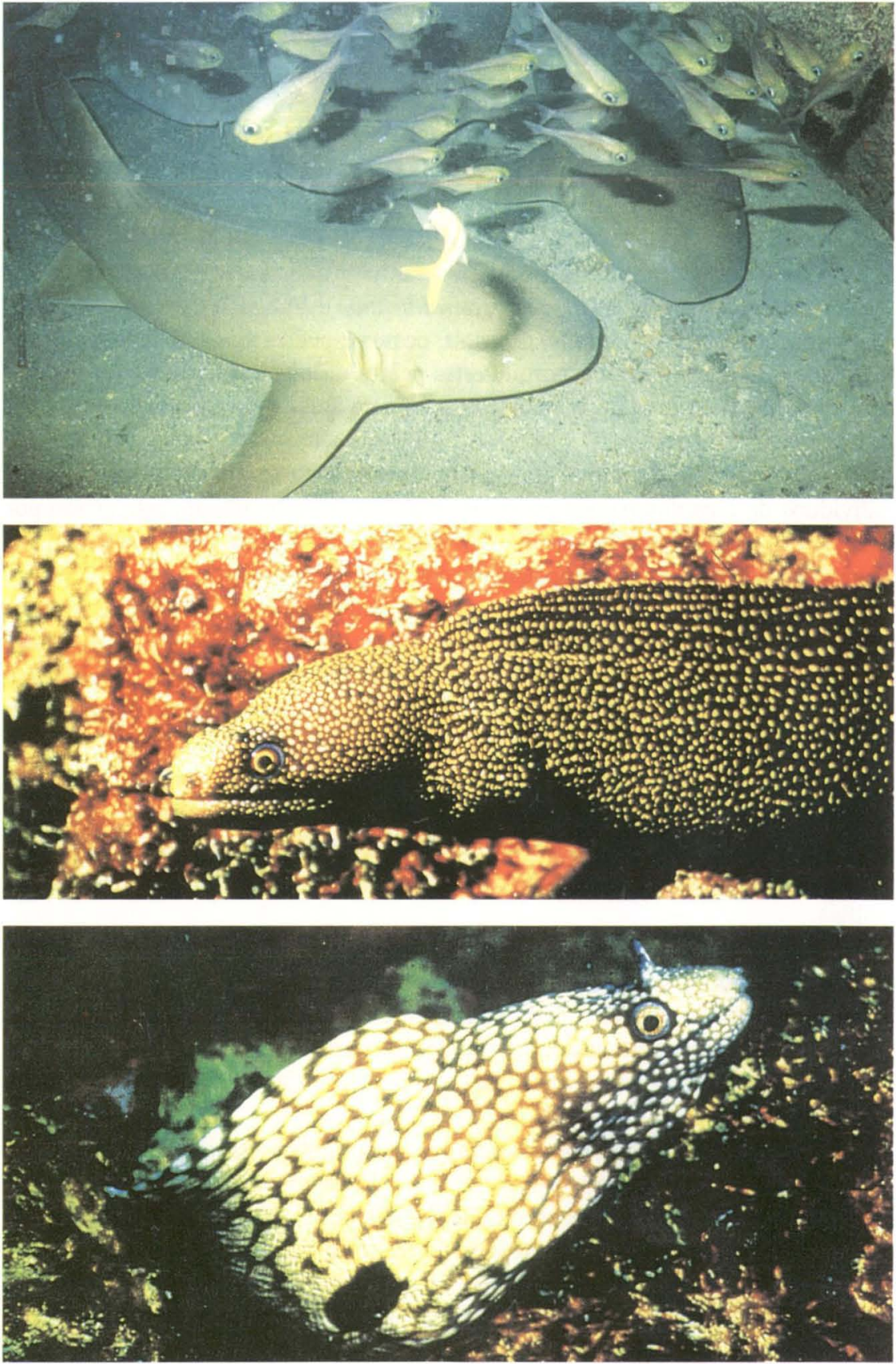

Fig. 2. (A) Ginglymostoma cirratum e Pempheris schomburgki, naufrágio do Vapor Bahia; (B) Gymnothorax miliaris, naufrágio do Queimado; (C) Muraena pavonina, naufrágio do Queimado. 
gramma gregoryi, Ulaema lefroyi (Goode, 1874), Eucinostomus gula (Cuvier, 1830), Haemulon chrysagyreum Günther, 1859, Haemulon macrostomum Günther, 1859, Equetus lanceolatus (Linnaeus, 1758), Sphyraena guachancho Cuvier, 1830, Sparisoma atomarium (Poey, 1861), Parablennius pilicornis (Cuvier, 1859) e Sphoeroides spengleri (Bloch, 1785).

Durante os mergulhos diurnos nos recifes situados em profundidades variando entre 6 e $20 \mathrm{~m}$, observou-se grandes cardumes de representantes do gênero Haemulon, principalmente Haemulon aurolineatum e Haemulon sp (Fig. 3b), mas, em mergulhos noturnos, nenhum exemplar dessas espécies foi observado. Segundo RANDALL (1967) isso ocorre porque estes peixes utilizam as construções recifais para proteção durante o dia, e à noite migram para ambientes adjacentes em busca de alimento.

No naufrágio do Vapor Bahia, observou-se uma agregação de cerca de 10 indivíduos de Gynglimostoma cirratum (Fig. 2a), comportamento este citado por Compagno (1984) para a espécie. Entretanto, nos demais pontos em que foi observada, a mesma estava representada por indivíduos isolados.

Entre os pontos amostrados, o naufrágio do Queimado apresentou o maior número de espécies da família Muraenidae representadas por Gymnothorax funebris, G.moringa, G. vicinus (a mais representativa numericamente), G. miliaris e Muraena pavonina, estas duas últimas (Fig. 2b,c) não sendo vistas em nenhum outro ponto visitado.

Em toda a área estudada a presença de peixes herbívoros é marcante, principalmente representantes das famílias Pomacentridae, Acanthuridae e Scaridae. Estas observações estão de acordo com SALE (1991) que indica os representantes dessas famílias como os herbívoros mais característicos de ambientes recifais.

Nos recifes mais rasos (1-6m de profundidade), à exemplo dos resultados obtidos por FERrEIRA et al. (1995), os pomacentrídeos Stegastes fuscus e S. variabilis foram as espécies mais comumente observadas. Ainda no grupo dos herbívoros, merecem destaque os acanturídeos Acanthurus chirurgus $\mathrm{e}$ A. coeruleus, que foram observados em cerca de $90 \%$ dos pontos visitados, independentemente da profundidade.

Exemplares de Chaetodon ocellatus, Malacanthus plumieri, Mycteroperca bonaci, Lutjanus analis, Lutjanus jocu e Trachinotus falcatus que tinham distribuição batimétrica conhecida até os 30m de profundidade (HUMANN 1995; LIESKE \& MYERS 1994), foram observados a 66m, no recife da Parede dos Dentões.

As novas ocorrências observadas, associadas às dificuldades encontradas na identificação específica precisa, indicam a necessidade de estudos sistemáticos adicionais nas áreas recifais da região, principalmente naquelas de maior profundidade. Além disso, também são necessários estudos populacionais com enfoque nas espécies explotadas. 

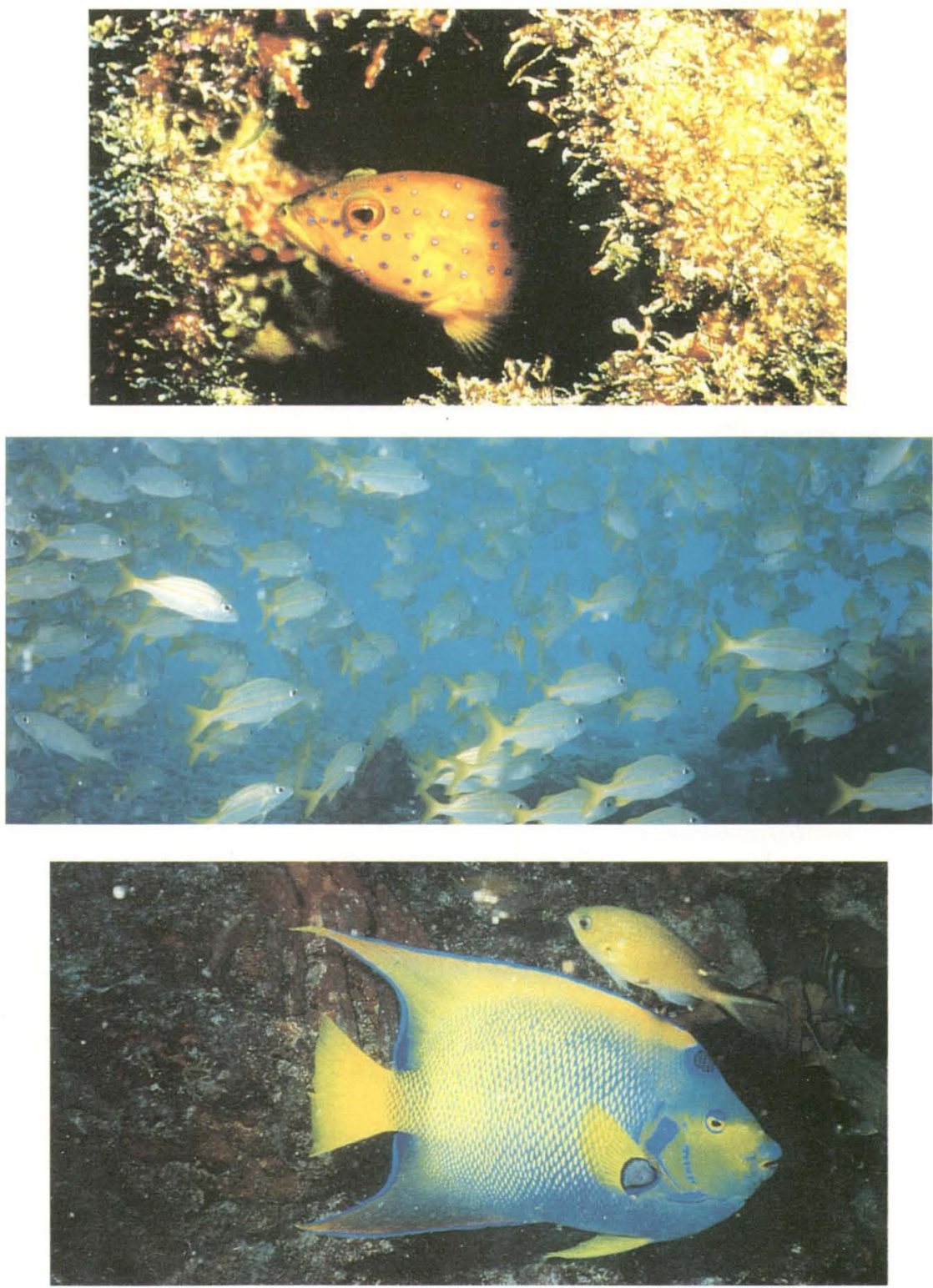

Fig. 3. (A) Cephalopholis fulva, naufrágio do Queimado; (B) Cardume de Haemulon sp., naufrágio do Queimado; (C) Holacanthus ciliaris e Chromis multilineata, recife do Taci de Cima. 


\section{Lista das espécies encontradas nos recifes da Paraíba}

Ginglymostomatidae

Ginglymostoma cirratum (Bonnaterre, 1788)

Carcharhinidae

Rhizoprionodon porosus (Poey, 1861)

Carcharhinus cf. perezi (Poey, 1876)

Sphyrnidae

Sphyrna tiburo (Linnaeus, 1758)

Torpedinidae

Narcine brasiliensis (Olfers, 1831)

Dasyatidae

Dasyatis americana Hildebrand \& Schroder, 1928

Dasyatis sp. $\left({ }^{*}\right)$

Myliobatidae

Aetobatus narinari (Euphrasen, 1790)

Mobulidae

Manta birostris (Donndorff, 1798) $\left({ }^{*}\right)$

Albulidae

Albula vulpes (Linnaeus, 1758)

Muraenidae

Echidna catenata (Bloch, 1795)

Enchelycore nigricans (Bonaterre, 1788)

Gymnothorax funebris Ranzani, 1840, UFPB 3350, 3489

Gymnothorax miliaris (Kaup, 1856) $(*)$

Gymnothorax moringa (Cuvier, 1829), UFPB 3344

Gymnothorax ocellatus (Agassiz, 1831)

Gymnothorax vicinus (Castelnau, 1855), UFPB 3490

Muraena pavonina (Richardson, 1844) $\left({ }^{*}\right)$

Ophichthidae

Ahlia egmontis (Jordan, 1844), UFPB 3336

Myrichthys ocellatus (Kaup, 1856), UFPB 3335

Ogcocephalidae

Ogcocephalus vespertilio (Linnaeus, 1758)

Gobiesocidae

Gobiesox strumosus Cope, 1870

Synodontidae

Synodus foetens (Linnaeus, 1758)

Batrachoididae

Thalassophryne nattereri Steindachner, 1876, UFPB 3338

Mugilidae

Mugil curema Cuvier \& Valenciennes, 1836

Revta bras. Zool. 15 (2): 553 - 566, 1998 
Mugil liza Valenciennes, 1836

Holocentridae

Holocentrus ascensionis (Osbeck, 1765), UFPB 3491

Myripristis jacobus Cuvier, 1829

Plectrypops retrospinis (Guichenot, 1855) $\left({ }^{*}\right)$

Dactylopteridae

Dactylopterus volitans (Linnaeus, 1758)

Syngnathidae

Hippocampus reidi Ginsburg, 1933

Scorpaenidae

Scorpaena plumieri Bloch, 1789, UFPB 3337

Triglidae

Prionotus punctatus (Bloch, 1797)

Serranidae

Alphestes afer (Bloch, 1793)

Cephalopholis fulva (Linnaeus, 1758), Fig. 3A

Diplectrum formosum (Linnaeus, 1766)

Epinephelus adscensionis (Osbeck, 1771)

Epinephelus guttatus (Linnaeus, 1758) (*)

Epinephelus itajara (Lichtenstein, 1822) $\left(^{*}\right)$

Mycteroperca bonaci (Poey, 1860) (*)

Rypticus saponaceus (Bloch \& Schneider, 1801) (*)

Rypticus subbifrenatus Gill, 1861, UFPB 3340 (*)

Serranus annularis (Günther, 1880) (*)

Serranus flaviventris (Cuvier \& Valenciennes, 1830)

Grammatidae

Gramma aff. loreto Poey, $1868\left(^{*}\right)$

Priacanthidae

Priacanthus arenatus Cuvier \& Valenciennes, 1829

Apogonidae

Apogon americanus Castelnau, 1855, UFPB 3359, 3492

Phaeoptyx pigmentaria (Poey, 1860), UFPB 3357, $3493\left({ }^{*}\right.$ )

Malacanthidae

Malacanthus plumieri (Bloch, 1786)

Echeneidae

Echeneis naucrates (Linnaeus, 1758)

Rachycentridae

Rachycentron canadum (Linnaeus, 1766) $\left({ }^{*}\right)$

Carangidae

Alectis ciliaris (Bloch, 1788) $\left(^{*}\right)$

Caranx hippos (Linnaeus, 1766) (*)

Caranx latus Agassiz, 1831 
Caranx lugubris Poey, $1860\left(^{*}\right)$

Carangoides bartholomaei Cuvier, 1833

Carangoides crysos (Mitchill, 1815) (*)

Elagatis bipinnulatus (Quoy \& Gaimard, 1824) (*)

Naucrates ductor (Linnaeus, 1758) (*)

Trachinotus falcatus (Linnaeus, 1758)

Trachinotus goodei Jordan \& Evermann, 1896

Selar crumenophthalmus (Bloch, 1793)

Selene vomer (Linnaeus, 1758)

Lutjanidae

Lutjanus analis (Cuvier \& Valenciennes, 1828)

Lutjanus apodus (Walbaum, 1792) (*)

Lutjanus cyanopterus (Cuvier, 1828) $\left({ }^{*}\right)$

Lutjanus jocu (Bloch \& Schneider, 1801)

Lutjanus synagris (Linnaeus, 1758)

Ocyurus chrysurus (Bloch, 1791)

Gerreidae

Eucinostomus argenteus Baird \& Girard, 1854

Eucinostomus melanopterus (Bleeker, 1863)

Haemulidae

Anisotremus moricandi (Ranzani, 1840)

Anisotremus surinamensis (Bloch, 1791), UFPB $3358\left(^{*}\right.$ )

Anisotremus virginicus (Linnaeus, 1758)

Haemulon aurolineatum Cuvier, 1829

Haemulon parra (Desmarest, 1823), UFPB $3498\left(^{*}\right)$

Haemulon plumieri (Lacépède, 1802)

Haemulon steindachneri (Jordan \& Gilbert, 1882), UFPB 3495

Haemulon sp., UFPB 3364, 3370 (*)

Orthopristis ruber (Cuvier \& Valenciennes, 1830)

Sparidae

Archosargus rhomboidalis (Linnaeus, 1758) $\left({ }^{*}\right)$

Calamus pennatula Guichenot, 1855

Polynemidae

Polydactylus virginicus (Linnaeus, 1758)

Sciaenidae

Odontoscion dentex (Cuvier, 1830)

Pareques acuminatus (Bloch \& Schneider, 1801), UFPB 3352, 3494

Umbrina coroides (Cuvier, 1830)

Mullidae

Mulloidichthys martinicus (Cuvier \& Valenciennes, 1829) $\left({ }^{*}\right.$ )

Pseudupeneus maculatus (Bloch, 1793) 
Pempheridae

Pempheris schomburgki Müller \& Troschel, $1848\left(^{*}\right)$

Chaetodontidae

Chaetodon ocellatus Bloch, 1787, UFPB 3355

Chaetodon striatus Linnaeus, 1758 (*)

Pomacanthidae

Holacanthus ciliaris (Linnaeus, 1758), Fig. 3C $(*)$

Holacanthus tricolor (Bloch, 1795) $(*)$

Pomacanthus arcuatus (Linnaeus, 1758) (*)

Pomacanthus paru (Bloch, 1787) (*)

Kyphosidae

Kyphosus sectatrix (Linnaeus, 1758)

Cirrhitidae

Amblycirrhitus pinos (Mowbray, 1927) $\left({ }^{*}\right)$

Pomacentridae

Abudefduf saxatilis (Linnaeus, 1758), UFPB 3346

Chromis multilineata (Guichenot, 1855), Fig. 3C (*)

Chromis flavicauda (Günther, 1880) (*)

Microspathodon chrysurus (Cuvier \& Valenciennes, 1830) $\left({ }^{*}\right.$ )

Stegastes fuscus (Cuvier \& Valenciennes, 1830), UFPB 3500

Stegastes aff dorsopunicans (Poey, 1860) (*)

Stegastes pictus (Castelnau, 1855) $\left(^{*}\right)$

Stegastes variabilis (Castelnau, 1855), UFPB 3353, 3499

Labridae

Bodianus pulchellus (Poey, 1860) (*)

Bodianus rufus (Linnaeus, 1758)

Clepticus sp. $(*)$

Halichoeres bivittatus (Bloch, 1791) (*)

Halichoeres cyanocephalus (Bloch, 1791) $\left({ }^{*}\right)$

Halichoeres maculipinna (Müller and Troschel, 1848)

Halichoeres poeyi (Steindachner, 1867), UFPB 3348

Halichoeres aff radiatus (Linnaeus, 1758), UFPB 3349

Hemipteronotus martinicensis Cuvier, 1839

Thalassoma noronhanum (Boulenger, 1888) $\left({ }^{*}\right)$

Scaridae

Cryptotomus roseus Cope, 1871

Scarus coelestinus Cuvier \& Valenciennes, 1839

Scarus sp., UFPB $3365\left(^{*}\right)$

Sparisoma chrysopterum (Bloch and Schneider, 1801) $\left({ }^{*}\right)$

Sparisoma radians (Cuvier \& Valenciennes, 1839)

Sparisoma rubripinne Cuvier \& Valenciennes, 1839, UFPB 3360, 3504

Sparisoma aff viride (Bonaterre, 1788) 
Trypterigiidae

Enneanectes altivelis Rosemblatt, 1960, UFPB 3367, 3501 (*)

Dactyloscopidae

Dactyloscopus tridigitatus Gill, 1859, UFPB 3339

Labrisomidae

Labrisomus kalisherae (Jordan, 1904), UFPB $3503\left({ }^{*}\right.$ )

Labrisomus nuchipinnis (Quoy \& Gaimard, 1824), UFPB 3497

Malacoctenus delalandii (Valenciennes, 1836), UFPB 3347, 3368, 3496

Malacoctenus aff. triangulatus Springer, 1958, UFPB 3794 (*)

Paraclinus nigripinnis (Steindachner, 1867), UFPB 3345

Blenniidae

Ophioblennius atlanticus (Cuvier \& Valenciennes, 1836), UFPB 3341, $3502\left({ }^{*}\right.$ )

Entomacrodus nigricans Gill, 1859, UFPB $3342(*)$

Scartella cristata (Linnaeus, 1758), UFPB $3361\left(^{*}\right)$

Gobiidae

Barbulifer ceuthoecus (Jordan \& Gilbert, 1884), UFPB 3343

Bathygobius soporator (Cuvier \& Valenciennes, 1837), UFPB 3356

Coryphopterus glaucofraenum Gill, 1863, UFPB 3369 (*)

Elacatinus figaro Sazima, Moura \& Rosa, 1996, UFPB $3366\left(^{*}\right)$

Gobionellus boleosoma (Jordan \& Gilbert, 1882)

Gobiosoma spilotum (Ginsburg, 1939), UFPB 3354

Ephippidae

Chaetodipterus faber (Broussonet, 1782)

Acanthuridae

Acanthurus bahianus Castelnau, 1855, UFPB $3505\left(^{*}\right)$

Acanthurus chirurgus (Bloch, 1787), UFPB 3363

Acanthurus coeruleus Bloch \& Schneider, 1801, UFPB 3362, $3507\left(^{*}\right)$

Sphyraenidae

Sphyraena barracuda (Walbaum, 1792) $(*)$

Sphyraena picudilla (Poey, 1860) (*)

Scombridae

Scomberomorus cavalla (Cuvier, 1829) (*)

Scomberomorus brasiliensis Collette, Russo \& Zavala-Camin, 1978

Bothidae

Bothus lunatus (Linnaeus, 1758) (*)

Achiridae

Achirus achirus (Linnaeus, 1758)

Balistidae

Balistes vetula Linnaeus, 1758

Canthidermis sufflamen (Mitchill, 1815) $\left(^{*}\right)$

Melichthys niger (Bloch, 1786) (*) 
Monacanthidae

Aluterus monoceros (Linnaeus, 1758) (*)

Cantherhines pullus (Ranzani, 1842) (*)

Ostraciidae

Acanthostracion polygonius Poey, $1876\left(^{*}\right)$

Acanthostracion quadricornis (Linnaeus, 1758)

Lactophrys trigonus (Linnaeus, 1758) (*)

Tetraodontidae

Sphoeroides testudineus (Linnaeus, 1758)

Diodontidae

Chilomycterus antillarum (Jordan \& Rutter, 1897)

Diodon holocanthus Linnaeus, 1758

Diodon hystrix Linnaeus, 1758 (*)

\section{REFERÊNCIAS BIBLIOGRÁFICAS}

BAKUS, G.J. 1994. Coral Reef Ecosystems. Rotterdam, A.A. Balkema, 232p.

BöHlke, J.E. \& C.C.G. Chaplin. 1993. Fishes of the Bahamas and Adjacent

Tropical Waters. Austin, University of Texas Press, $2^{\text {nd }}$ ed., $771 \mathrm{p}$.

BREwER, R. 1994. The Science of Ecology. New York, Saunders College Publishing, $2^{\text {nd }}$ ed., $773 \mathrm{p}$.

Briggs, J.C. 1974. Marine Zoogeography. New York, McGraw-Hill Book Co., $475 \mathrm{p}$.

CHoU, L.M. 1991. Artificial reefs in Singapore: development potential and constrains, p.47-52. In: L.S. CHIA \& L.M. CHOU (Eds) Urban coastal area management: the experience of Singapore. ICLARM Conference Proceedings 25, $128 \mathrm{p}$.

Compagno, L.J.V. 1984. FAO species catalogue. Vol 4. Sharks of the world. An annotated and illustrated catalogue of sharks species known to date. Part 1. Hexanchiformes to Lamniformes. FAO Fish. Synop. 4 (125): 1-249.

FERrEIRA, B.P.; M. MAIDA \& A.E.T. SouzA. 1995. Levantamento inicial das comunidades de peixes recifais da região de Tamandaré - PE. Bol. Tecn. Cient. CEPENE 3 (1): 211-230.

Humann, P. 1995. Reef Fish Identification - Florida, Caribbean, Bahamas. Jacksonville, New World Publications, $2^{\text {nd }}$ ed., $396 \mathrm{p}$.

KEMPF, M. 1970. Notes on the benthic bionomy of the N-NE Brazilian shelf. Mar. Biol. 5: 213-224.

Koike, J. \& D.S. Guedes. 1981. Peixes dos arrecifes de Pernambuco e Estados vizinhos. Anais do III Encontro de Zoologia do Nordeste, Recife: 35-82.

LEÃO, Z.M.A.N. 1986. Guia para Identificação dos Corais do Brasil. Salvador, Universidade Federal da Bahia, Programa de Pesquisa e Pós-Graduação em Geofísica, $57 \mathrm{p}$.

Lieske, E. \& R. Myers. 1994. Collins Pocket Guide to Coral Reef Fishes of the Caribbean, Indian Ocean and Pacific. London, Harper Collins Publishers, 400 p. 
Menezes, N.A. \& J.L. Figueiredo. 1985. Manual de Peixes Marinhos do Sudeste do Brasil. Teleostei (4). São Paulo, Museu de Zoologia, Universidade de São Paulo, 96p.

MOYLE, P.B. \& J.J. CECH. 1996. Fishes: An Introduction to Ichthyology. New Jersey, Prentice-Hall Inc., $3^{\text {rd }}$ ed., 590 p.

NeLson, J.S. 1994. Fishes of the World. New York, John Wiley \& Sons Inc., $3^{\text {rd }}$ ed., 600 p.

PotTs, T.A. \& A.W. HuLBERT. 1994. Structural influences of artificial and natural habitats on fish aggregations in Onslow Bay, North Carolina. Bull. Mar. Sci. 55 (2): 609-622.

Ramos, R.T.C. 1994. Análise da composição e distribuição da fauna de peixes demersais da plataforma continental da Paraíba e estados vizinhos. Revta Nordest. Biol., João Pessoa, 9 (1): 1-30.

Randall, J.E. 1967. Food habits of reef fishes of West Indies. Stud. Trop. Oceanogr. 5: 665-847.

. 1968. Caribbean Reef Fishes. Hong Kong, T.F.H. Publications, 318p.

RosA, R.S. 1980. Lista sistemática de peixes marinhos da Paraíba (Brasil). Revta Nordest. Biol., João Pessoa, 3 (2): 205-226.

RosA, R.S \& R.L. MouRA. 1997. Visual assessment of reef fish community structure in the Atol das Rocas Biological Reserve, off Northeastern Brazil. Proc. $8^{\text {th }}$ Int. Coral Reef Sym. 1: 983-986.

RosA, R.S.; I.L. RosA \& L.A. RocHA. 1997. Diversidade da ictiofauna de poças de maré da praia do Cabo Branco, João Pessoa, Paraíba, Brasil. Revta bras. Zool. 14 (1): 201-212.

RouX, C. 1973. Téléostéens du Plateau Continental Brésilien, Compagne de la Calypso au large des cotes Atlantiques de L'Amérique du Sud (1961-1962). Premiere partie, 189p.

Sale, P.F. 1991. The Ecology of Fishes on Coral Reefs. San Diego, Academic Press, 754p.

SALE, P.F.; G.E. Forrester \& P.S. LeVIN. 1994. Reef Fish Management. National Geographic Research \& Exploration 10 (2): 224-235.

STARKS, E.C. 1913. The fishes of the Stanford expedition to Brazil. Leland Stanford Univ. Publ., Univ. Ser.: 1-77.

Wells, S. \& N. HANNA. 1992. The Greenpeace book of Coral Reefs. London, Blandford, 160p.

White, A.T.; L.Z. HAle; Y. Renard \& L. CORTESI. 1994. Collaborative and Community-Based Management of Coral Reefs. West Hartford, Kumarian Press, 130p.

Recebido em 18.IV.1997; aceito em 25.VI.1998. 